\title{
Study on the management of patients' choice of medical institutions based on DEMATEL method
}

\author{
Chen Peng ${ }^{1, a}$, Li Yueyu ${ }^{2, b}$
}

${ }^{1}$ Business school of Sichuan University, Chengdu, Sichuan, China

${ }^{2}$ Business school of Sichuan University, Chengdu, Sichuan, China

a597488057@qq.com, bliyueyu@scu.edu.cn

${ }^{*}$ Chen Peng

Keywords: Selection of medical institutions, Influencing factors, DEMATEL method.

\begin{abstract}
The choice of medical institution is a complicated decision-making process, which is influenced by many factors. In this paper, we systematically analyzed the influence factors of patients to choose medical institutions from three aspects: patients, medical institutions and government, and used DEMATEL method to analyze the causal relationship of the factors influencing patients' choice of medical institutions. Meanwhile, according to the quantitative results, we made causal classification and importance ranking of the influencing factors of patients' choice of medical institutions, and found out the key influencing factors. We expect to provide scientific basis for managing patients' choice of medical institutions and reforming China's medical system.
\end{abstract}

\section{基于DEMATEL法的患者选择医疗机构管理研究 \\ 陈鹏 $1, \mathrm{a}$, 李跃宇 $2, \mathrm{~b}$ \\ 1 四川大学商学院, 成都, 四川, 中国 \\ 2 四川大学商学院, 成都, 四川, 中国 \\ a597488057@qq.com, bliyueyu@scu.edu.cn \\ *陈鹏}

关键词: 医疗机构选择; 影响因素; DEMATEL方法

摘要. 患者选择医疗机构是一个复杂的决策过程, 受到很多因素的影响。本文从供方、需方 和政府三个方面对患者选择医疗机构的影响因素进行系统分析, 并利用 DEMATEL 方法对影 响患者选择医疗机构的因素进行因果关系分析, 同时根据量化结果对影响因素进行因果分类 和重要程度排序, 找出影响患者选择医疗机构的关键因素, 为管理患者选择医疗机构和改革 我国医疗体制提供科学依据。

\section{1. 引言}

患者选择医疗机构的影响因素是指患病后需要寻求医疗服务时, 影响患者选择某特定医 疗机构就诊的因素 ${ }^{[1]}$ 。患者选择医疗机构是一个复杂的决策过程, 受到很多因素的影响, 其 中包括患者自身的因素, 医疗机构的因素以及我国医疗卫生政策的因素, 这些因素综合作用 在一起使得患者在选择医疗机构时十分困惑。如果患者没有选择与其病情相匹配的医疗机构, 就很可能达不到理想的治疗效果, 并造成医疗资源的浪费。准确了解患者选择医疗机构的影 
响因素, 有助于引导患者合理就医, 有助于提高医院管理水平, 有助于制定合理的医疗卫生 政策。由此可见，研究患者选择医疗机构的影响因素具有重要意义。

影响患者选择医疗机构的因素有很多, 如交通便利程度、疾病严重程度、机构诊疗水平、 机构规模、服务态度等, 在影响患者选择医疗机构的众多因素中, 哪些是直接影响因素? 哪 些是通过其他因素影响患者选择医疗机构的间接因素? 哪些是影响患者选择医疗机构的关键 因素? 这正是本文要解决的主要问题。

\section{2. 患者选择医疗机构影响因素体系的构建}

患者选择医疗机构受到很多因素的影响, 本文将这些影响因素归纳为供方、需方和政府 三个方面。

\section{1 供方影响因素}

供方影响因素即医疗机构对患者选择医疗机构的影响因素, 主要包括交通便利程度、机 构诊疗水平、医疗服务价格、报销比例和服务态度等。

我国西部偏远农村地区交通不便, 患者在选择医疗机构就诊时不仅考虑医疗机构的诊疗 水平, 还会考虑到交通便利程度这一影响因素, 因为长途异地就医不仅会增加费用的支出, 还有可能延误病情。钱东福 ${ }^{2}$ ]指出, 医疗机构的地理可及性将直接影响农村居民门诊就医时 对于医疗机构的选择。除此之外, 患者在选择医疗机构时还会特别重视医疗机构的诊疗水平、 服务价格和报销比例。毛柳东等 ${ }^{[3]}$ 发现, 患者选择三级综合医院主要是因为信任三级综合医 院的诊疗水平。于长久 ${ }^{[4]}$ 指出, 农民就医时不选择乡镇医院主要是因为乡镇医院的医生水平 差, 医疗设施简陃。张黎 ${ }^{[5]}$ 等发现, 低收入的患者在选择医疗机构时, 首先考虑的是医疗服 务价格和报销比例因素。

\section{2 需方影响因素}

需方影响因素即患者自身情况对其选择医疗机构的影响因素, 主要包括年龄、受教育程 度、疾病严重程度、经济状况、是否参与医疗保险和是否有熟悉的医生等。

不同年龄段的患者在选择医疗机构时具有不同的偏好。老年患者在选择医疗机构时倾向 于社区医院、村卫生室或乡镇卫生院, 因为他们更加注重就医的便利程度; 其他年龄段的患 者选择医疗机构时更加关注医疗机构的诊疗水平、服务态度等 ${ }^{[5]}$ 。张黎等 ${ }^{[6]}$ 发现, 受教育程度 高的患者在选择医疗机构时更加关注医疗机构的知名度, 而受教育程度低的患者在选择医疗 机构时主要是依据以往的就医经验。一般来说, 受教育程度高的患者能比较准确的感知自身 的病情, 他们在选择医疗机构时更加注重医疗机构和自身病情的匹配程度, 不会盲目地选择 医疗机构。另外, 医疗保险对于患者选择医疗机构也有比较显著的影响, 但其影响比较复杂, 主要体现在是否参与医疗保险, 以及医疗保险的保障能力。

\section{3 政府影响因素}

政府对患者选择医疗机构的影响主要体现在医疗卫生制度上, 其中影响最为显著的即分 级诊疗制度。

为引导居民合理就医, 充分利用各级医疗资源, 2015年国家出台了《关于推进分级诊疗 制度建设的指导意见》。分级诊疗是指将疾病按照轻、重、缓、急及治疗的难易程度进行分 级, 要求不同级别的医疗机构承担不同等级疾病的治疗, 明确分工, 逐步实现合理就医 ${ }^{[7]}$ 。

通过以上分析, 我们不难发现影响患者选择医疗机构的各因素并不是完全独立的, 它们 是相互依存, 相互影响的。例如, “医疗服务价格” 因素, 直接影响患者选择医疗机构, 同 时也受 “机构诊疗水平” 的影响。患者选择医疗机构的影响因素体系如表1所示。 
表1＼cjkstart患者选择医疗机构的影响因素体系

\begin{tabular}{|c|c|c|}
\hline 方面 & 影响因素 & 编号 \\
\hline \multirow{4}{*}{ 供方 } & 交通便利程度 & $\mathrm{S}_{1}$ \\
\cline { 2 - 3 } & 机构诊疗水平 & $\mathrm{S}_{2}$ \\
\cline { 2 - 3 } & 医疗服务价格 & $\mathrm{S}_{3}$ \\
\cline { 2 - 3 } & 报销比例 & $\mathrm{S}_{4}$ \\
\cline { 2 - 3 } & 服务态度 & $\mathrm{S}_{5}$ \\
\hline \multirow{4}{*}{ 需方 } & 年龄 & $\mathrm{S}_{6}$ \\
\cline { 2 - 3 } & 受教育程度 & $\mathrm{S}_{7}$ \\
\cline { 2 - 3 } & 疾病严重程度 & $\mathrm{S}_{8}$ \\
\cline { 2 - 3 } & 经济状况 & $\mathrm{S}_{9}$ \\
\cline { 2 - 3 } & 是否有熟悉的医生 & $\mathrm{S}_{10}$ \\
\cline { 2 - 3 } & 是否参与医疗保险 & $\mathrm{S}_{11}$ \\
\hline 政府 & 分级诊疗制度 & $\mathrm{S}_{12}$ \\
\hline
\end{tabular}

\section{3. 基于DEMATEL方法的患者选择医疗机构影响因素分析}

DEMATEL方法通过系统中各因素之间的逻辑关系和直接影响矩阵可计算出每个因素的 原因度与中心度，从而确定因素间的因果关系和每个因素在系统中的地位。基于DEMATEL 方法的患者选择医疗机构影响因素分析的具体步骤如下:

步骤一：确定影响因素

将患者选择医疗机构的影响因素分别记为 $\mathrm{S}_{1}, \mathrm{~S}_{2}, \ldots \ldots, \mathrm{S}_{12}$ 。

步骤二：建立初始直接影响矩阵 $\boldsymbol{A}=\left[\mathrm{a}_{\mathrm{ij}}\right]_{\mathrm{n} \times \mathrm{n}}$

采用 $0 \sim 4$ 标度对患者选择医疗机构影响因素间的直接影响程度进行打分，即可得到以矩 阵形式表示的直接影响矩阵 $\boldsymbol{A}=\left[\mathrm{a}_{\mathrm{ij}}\right]_{\mathrm{n} \times \mathrm{n}}$, 其中 $\mathrm{a}_{\mathrm{ij}}(i=1,2, \ldots, n ; j=1,2, \ldots, n)$ 表示因素 $\mathrm{S}_{\mathrm{i}}$ 对因素 $\mathrm{S}_{\mathrm{j}}$ 的直 接影响程度, $i=j$ 时, $\mathrm{a}_{\mathrm{ij}}=0$ 。数值 $\mathrm{a}_{\mathrm{ij}}$ 的定义和解释见表 2 。

$$
\boldsymbol{A}=\left[\begin{array}{cccc}
0 & a_{12} & \cdots & a_{1 n} \\
a_{21} & 0 & \cdots & a_{2 n} \\
\vdots & \vdots & \cdots & \vdots \\
a_{n 1} & a_{n 2} & \cdots & 0
\end{array}\right]
$$

表2 $\mathrm{a}_{\mathrm{ij}}$ 的定义和解释

\begin{tabular}{|c|c|c|}
\hline $\mathbf{a}_{\mathbf{i j}}$ & 定义 & 解释 \\
\hline 0 & 没有直接影响 & 所有人都认为 $\mathrm{s}_{\mathrm{i}}$ 对 $\mathrm{s}_{\mathrm{j}}$ 没有直接影响 \\
\hline 1 & 很弱的直接影响 & 有 $0 \sim 30 \%$ 的人认为 $\mathrm{s}_{\mathrm{i}}$ 对 $\mathrm{s}_{\mathrm{j}}$ 有直接影响 \\
\hline 2 & 中等的直接影响 & 有 $30 \% \sim 60 \%$ 的人认为 $\mathrm{s}_{\mathrm{i}}$ 对 $\mathrm{s}_{\mathrm{j}}$ 有直接影响 \\
\hline 3 & 较强的直接影响 & 有 $60 \% \sim 90 \%$ 的人认为 $\mathrm{s}_{\mathrm{i}}$ 对 $\mathrm{s}_{\mathrm{j}}$ 有直接影响 \\
\hline 4 & 很强的直接影响 & 有 $90 \%$ 以上的人认为 $\mathrm{s}_{\mathrm{i}}$ 对 $\mathrm{s}_{\mathrm{j}}$ 有直接影响 \\
\hline
\end{tabular}

步骤三: 建立标准化直接影响矩阵 $N$

将矩阵 $\boldsymbol{A}$ 中元素按行相加, 设行和最大值为 $\max$, 则标准化直接影响矩阵

步骤四：计算综合影响矩阵 $\boldsymbol{T}$

$$
\boldsymbol{N}=\frac{A}{\max }
$$

综合影响矩阵 $\boldsymbol{T}=\boldsymbol{N}+N^{2}+N^{3}+\cdots+N^{n}$, 当n充分大时, 可以用 $\boldsymbol{N}(\boldsymbol{E}-\boldsymbol{N})^{-1}$ 近似表示 $\boldsymbol{T}$, 其中 $\boldsymbol{E}$ 为单位矩阵。 $\mathrm{t}_{\mathrm{ij}}$ 表示因素 $\mathrm{s}_{\mathrm{i}}$ 对因素 $\mathrm{S}_{\mathrm{j}}$ 的直接影响和间接影响的程度。

步骤五: 计算各因素的影响度 $\mathrm{f}_{\mathrm{i}}$ 和被影响度 $\mathrm{e}_{\mathrm{i}}$

$$
\boldsymbol{T}=\boldsymbol{N}(E-N)^{-1}
$$


将矩阵 $\boldsymbol{T}$ 中元素按行相加得到相应因素的影响度 $\mathrm{f}_{\mathrm{i}}$ （即各行对应因素对所有其他因素的综 合影响值）, 将矩阵 $\boldsymbol{T}$ 中元素按列相加得到相应因素的被影响度 $\mathrm{e}_{\mathrm{i}}$ （即各列对应因素受到所有 其他因素的综合影响值）。计算公式如下:

$$
\begin{aligned}
& f_{i}=\sum_{j=1}^{n} t_{i j},(i=1,2, \cdots, n) \\
& e_{i}=\sum_{i=1}^{n} t_{i j},(j=1,2, \cdots, n)
\end{aligned}
$$

步骤六: 计算各因素的中心度 $\mathrm{m}_{\mathrm{i}}$ 和原因度 $\mathrm{n}_{\mathrm{i}}$

各因素的影响度和被影响度相加得到其中心度 $m_{i}, m_{i}$ 表示该因素在所有因素中的位置及 其所起作用的大小。各因素的影响度和被影响度相减得到其原因度 $\mathrm{n}_{\mathrm{i}}$, 其中 $\mathrm{n}_{\mathrm{i}}>0$ 的因素是原 因因素，原因因素表示该因素对其他因素影响大，且不易被改变；其中 $\mathrm{n}_{\mathrm{i}}<0$ 的因素是结果因 素, 结果因素表示该因素受其他因素影响大, 并且比较容易被改变。计算公式如下:

$$
\begin{aligned}
m_{i} & =f_{i}+e_{i},(i=1,2, \cdots, n) \\
n_{i} & =f_{i}-e_{i},(i=1,2, \cdots, n)
\end{aligned}
$$

表3 患者选择医疗机构影响因素的初始直接影响矩阵 $\boldsymbol{A}$

\begin{tabular}{ccccccccccccc}
\hline No. & $\mathrm{S}_{1}$ & $\mathrm{~S}_{2}$ & $\mathrm{~S}_{3}$ & $\mathrm{~S}_{4}$ & $\mathrm{~S}_{5}$ & $\mathrm{~S}_{6}$ & $\mathrm{~S}_{7}$ & $\mathrm{~S}_{8}$ & $\mathrm{~S}_{9}$ & $\mathrm{~S}_{10}$ & $\mathrm{~S}_{11}$ & $\mathrm{~S}_{12}$ \\
$\mathrm{~S}_{1}$ & 0 & 0 & 0 & 0 & 0 & 0 & 0 & 0 & 0 & 0 & 0 & 0 \\
$\mathrm{~S}_{2}$ & 0 & 0 & 4 & 0 & 0 & 0 & 0 & 3 & 0 & 0 & 0 & 1 \\
$\mathrm{~S}_{3}$ & 0 & 4 & 0 & 0 & 2 & 0 & 0 & 0 & 0 & 0 & 0 & 1 \\
$\mathrm{~S}_{4}$ & 3 & 0 & 0 & 0 & 0 & 0 & 0 & 0 & 0 & 0 & 0 & 0 \\
$\mathrm{~S}_{5}$ & 3 & 0 & 2 & 0 & 0 & 0 & 0 & 0 & 0 & 1 & 0 & 0 \\
$\mathrm{~S}_{6}$ & 3 & 0 & 0 & 1 & 0 & 0 & 2 & 1 & 2 & 3 & 2 & 0 \\
$\mathrm{~S}_{7}$ & 3 & 0 & 0 & 0 & 0 & 0 & 0 & 0 & 4 & 4 & 2 & 0 \\
$\mathrm{~S}_{8}$ & 4 & 0 & 0 & 4 & 0 & 2 & 0 & 0 & 4 & 0 & 0 & 0 \\
$\mathrm{~S}_{9}$ & 3 & 0 & 0 & 0 & 0 & 0 & 2 & 0 & 0 & 3 & 2 & 0 \\
$\mathrm{~S}_{10}$ & 0 & 4 & 0 & 0 & 3 & 0 & 0 & 0 & 0 & 0 & 0 & 0 \\
$\mathrm{~S}_{11}$ & 0 & 0 & 0 & 4 & 0 & 0 & 0 & 0 & 0 & 0 & 0 & 1 \\
$\mathrm{~S}_{12}$ & 0 & 3 & 2 & 4 & 0 & 0 & 0 & 0 & 0 & 0 & 3 & 0 \\
\hline
\end{tabular}

表4 患者选择医疗机构影响因素的综合影响矩阵 $\boldsymbol{T}$

\begin{tabular}{ccccccccccccc}
\hline No. & $\mathrm{S}_{1}$ & $\mathrm{~S}_{2}$ & $\mathrm{~S}_{3}$ & $\mathrm{~S}_{4}$ & $\mathrm{~S}_{5}$ & $\mathrm{~S}_{6}$ & $\mathrm{~S}_{7}$ & $\mathrm{~S}_{8}$ & $\mathrm{~S}_{9}$ & $\mathrm{~S}_{10}$ & $\mathrm{~S}_{11}$ & $\mathrm{~S}_{12}$ \\
$\mathrm{~S}_{1}$ & 0 & 0 & 0 & 0 & 0 & 0 & 0 & 0 & 0 & 0 & 0 & 0 \\
$\mathrm{~S}_{2}$ & 0.1350 & 0.1320 & 0.3470 & 0.1156 & 0.0567 & 0.0350 & 0.0164 & 0.2451 & 0.0797 & 0.0333 & 0.0420 & 0.1086 \\
$\mathrm{~S}_{3}$ & 0.0800 & 0.3554 & 0.1410 & 0.0623 & 0.1675 & 0.0110 & 0.0052 & 0.0770 & 0.0250 & 0.0212 & 0.0292 & 0.1090 \\
$\mathrm{~S}_{4}$ & 0.2143 & 0 & 0 & 0 & 0 & 0 & 0 & 0 & 0 & 0 & 0 & 0 \\
$\mathrm{~S}_{5}$ & 0.2320 & 0.0750 & 0.1727 & 0.0114 & 0.0410 & 0.0023 & 0.0011 & 0.0162 & 0.0053 & 0.0763 & 0.0051 & 0.0181 \\
$\mathrm{~S}_{6}$ & 0.3821 & 0.1104 & 0.0460 & 0.1666 & 0.0753 & 0.0137 & 0.1766 & 0.0961 & 0.2227 & 0.3208 & 0.2074 & 0.0260 \\
$\mathrm{~S}_{7}$ & 0.3325 & 0.1269 & 0.0526 & 0.0728 & 0.0871 & 0.0039 & 0.0444 & 0.0275 & 0.3068 & 0.3712 & 0.1994 & 0.0271 \\
$\mathrm{~S}_{8}$ & 0.4846 & 0.0426 & 0.0178 & 0.3274 & 0.0291 & 0.1457 & 0.0682 & 0.0195 & 0.3316 & 0.1238 & 0.0801 & 0.0100 \\
$\mathrm{~S}_{9}$ & 0.2906 & 0.0939 & 0.0391 & 0.0628 & 0.0641 & 0.0029 & 0.1503 & 0.0203 & 0.0492 & 0.2730 & 0.1765 & 0.0221 \\
$\mathrm{~S}_{10}$ & 0.0883 & 0.3395 & 0.1362 & 0.0355 & 0.2393 & 0.0105 & 0.0049 & 0.0735 & 0.0239 & 0.0259 & 0.0131 & 0.0349 \\
$\mathrm{~S}_{11}$ & 0.0696 & 0.0213 & 0.0172 & 0.3133 & 0.0026 & 0.0007 & 0.0003 & 0.0046 & 0.0015 & 0.0007 & 0.0165 & 0.0754 \\
$\mathrm{~S}_{12}$ & 0.1165 & 0.2979 & 0.2411 & 0.3865 & 0.0367 & 0.0092 & 0.0043 & 0.0645 & 0.0210 & 0.0103 & 0.2310 & 0.0550 \\
\hline
\end{tabular}


表5＼cjkstart患者选择医疗机构影响因素的综合影响关系

\begin{tabular}{ccccc}
\hline 影响因素 & 影响度 & 被影响度 & 中心度 & 原因度 \\
\hline $\mathrm{S}_{1}$ & 0 & 2.4255 & 2.4255 & -2.4255 \\
$\mathrm{~S}_{2}$ & 1.3464 & 1.5948 & 2.9412 & -0.2484 \\
$\mathrm{~S}_{3}$ & 1.0838 & 1.2107 & 2.2945 & -0.1270 \\
$\mathrm{~S}_{4}$ & 0.2143 & 1.5541 & 1.7684 & -1.3398 \\
$\mathrm{~S}_{5}$ & 0.6566 & 0.7994 & 1.4560 & -0.1427 \\
$\mathrm{~S}_{6}$ & 1.8437 & 0.2349 & 2.0786 & 1.6088 \\
$\mathrm{~S}_{7}$ & 1.6520 & 0.4717 & 2.1237 & 1.1804 \\
$\mathrm{~S}_{8}$ & 1.6803 & 0.644235 & 2.3245 & 1.0360 \\
$\mathrm{~S}_{9}$ & 1.2448 & 1.0667 & 2.3115 & 0.1782 \\
$\mathrm{~S}_{10}$ & 1.0254 & 1.2565 & 2.2819 & -0.2311 \\
$\mathrm{~S}_{11}$ & 0.5237 & 1.0003 & 1.5240 & -0.4767 \\
$\mathrm{~S}_{12}$ & 1.4739 & 0.4861 & 1.9601 & 0.9878 \\
\hline
\end{tabular}

\section{4. 案例研究}

\section{1 影响因素识别}

本文采用文献研究法和问卷调查法分析、总结患者选择医疗机构的影响因素, 最终从供 方、需方和政府三个方面构建出患者选择医疗机构影响因素体系，见表1。

\section{2 数据收集与处理}

本文选取不同级别医疗机构的患者和患者就医行为研究领域的专家学者（主要是指在国 内权威期刊上公开发表过有关患者就医行为方面文章的本校学者）为调查对象, 采用问卷调 查法对患者选择医疗机构影响因素间的直接影响程度进行 $0 \sim 4$ 分的打分。经筛选, 得到有效 问卷100份, 其中 60 份来自不同级别医疗机构的患者, 40份来自患者就医行为研究领域的专家 学者。

根据前述基于DEMATEL方法的患者选择医疗机构影响因素分析步骤二，对回收的100份 有效问卷进行统计分析后得到患者选择医疗机构影响因素的初始直接影响矩阵 $\boldsymbol{A}$, 见表 3 。然 后, 运用EXCEL, 将矩阵 $\boldsymbol{A}$ 中元素按行相加, 找出行和最大值max, 依据公式 (2) 计算出标 准化直接影响矩阵 $\boldsymbol{N}$, 再依据公式 (3) 计算出综合影响矩阵 $\boldsymbol{T}$, 见表 4 。再根据公式 (4), (5),

（6），（7）计算出各影响因素的影响度、被影响度、中心度和原因度, 从而得到各影响因 素的综合影响关系，见表5。

\section{3 结果分析}

\subsection{1 原因度分析}

根据原因度的正负可以将患者选择医疗机构的影响因素分为原因因素和结果因素。原因 因素 (原因度为正数) 不仅对患者选择医疗机构有显著影响, 还对其他因素产生影响, 并且 该因素不易被改变, 其原因度越大, 影响程度越大, 越难被改变。由综合影响关系（表6）可 以看出, 影响患者选择医疗机构的原因因素主要有: 年龄 $\left(\mathrm{S}_{6}\right)$ 、受教育程度 $\left(\mathrm{S}_{7}\right)$ 、疾病严 重程度 $\left(\mathrm{S}_{8}\right)$ 和分级诊疗制度 $\left(\mathrm{S}_{12}\right)$ 。因此, 患者应该综合考虑自身的情况, 理性地选择和 自身病情相匹配的医疗机构, 不要盲目地迷信高等级的大型综合医疗机构。政府应该继续完 善分级诊疗制度, 通过差异化医疗服务价格、差异化报销比例等措施科学、合理地分流患者, 以充分发挥各级医疗机构的作用，并不断加强基层医疗机构的建设。

结果因素 (原因度为负数) 是受原因因素的影响而间接影响患者选择医疗机构的主要因 素, 结果因素易受外界影响而发生改变。由综合影响关系 (表6) 可以看出, 影响患者选择医 
疗机构的结果因素主要有: 交通便利程度 $\left(\mathrm{S}_{1}\right)$ 、报销比例 $\left(\mathrm{S}_{4}\right)$ 、是否参与医疗保险 $\left(\mathrm{S}_{11}\right)$ 和医疗服务价格 $\left(\mathrm{S}_{3}\right)$ 。结果因素受原因因素的影响而间接影响患者选择医疗机构, 所以我 们应该将主要精力放在原因因素上, 原因因素解决之后, 结果因素自然而然也就解决了。如 报销比例因素，可以通过实施、完善分级诊疗制度来调整各级医疗机构的报销比例。

\subsection{2 中心度分析}

中心度表示该因素在所有因素中的位置及其所起作用的大小，中心度越大表示该因素对 患者选择医疗机构的影响越大, 中心度越小表示该因素对患者选择医疗机构的影响越小。由 综合影响关系 (表6) 可以看出, 影响患者选择医疗机构的关键因素有: 机构诊疗水平 $\left(\mathrm{S}_{2}\right)$ 、 交通便利程度 $\left(\mathrm{S}_{1}\right)$ 、经济状况 $\left(\mathrm{S}_{9}\right)$ 、疾病严重程度 $\left(\mathrm{S}_{8}\right)$ 、医疗服务价格 $\left(\mathrm{S}_{3}\right)$ 、受教育 程度 $\left(S_{7}\right)$ 、分级诊疗制度 $\left(S_{12}\right)$ 、报销比例 $\left(S_{4}\right)$ 等。针对以上关键因素, 可以从以下几个 方面考虑: 第一, 加强健康教育和政策宣传。可以由基层医疗机构定期组织居民开展健康教 育和政策宣传活动, 引导居民树立正确的健康观和就医习惯, 引导患者合理地选择医疗机构, 避免基层医疗资源的浪费, 同时也缓解大型医疗机构的负担。第二, 加强医疗队伍建设, 提 升诊疗水平。医院应该注重提升业务人员的技能水平及职业素养, 争取为患者提供高质量的 医疗服务, 从而提升医院自身的实力。第三, 减轻患者就医负担, 加强基层医疗机构建设。 政府在通过实施差异化医疗服务价格、差异化报销比例以合理分流患者的同时, 也要加大对 医疗卫生事业的财政投入，适当降低医疗服务价格，扩大医疗保障范围，提高报销比例，从 而减轻患者的就医负担。同时也应该加强基层医疗机构建设, 完善必要的医疗设施, 引进和 培养专业技术人员, 提升基层医疗机构的服务质量, 从而更好地推行基层首诊, 提高基层医 疗资源的利用率。

\section{5. 结论}

本文利用DEMATEL方法对患者选择医疗机构的影响因素进行了量化分析, 分析结果显 示: 机构诊疗水平、经济状况、疾病严重程度、医疗服务价格、分级诊疗制度、报销比例是 影响患者选择医疗机构的关键因素。因此, 政府在引导患者合理选择医疗机构以及改革医疗 体制时应首先考虑以上关键影响因素, 然后再考虑其他影响因素。

\section{References}

[1] Wei Min, The influence factors of patients to choose hospitals analysis, Anhui medical University, 2014.

[2] Qian Dongfu, Research on medical choice behavior of rural residents in Gansu province, Shandong University, 2008.

[3] Mao Liudong, Chen Yuhua, Study on the behavior of patients with chronic diseases, utilization of community health services and influencing factors, Journal of Gannan Medical College, vol.37, pp.283-285, 2017.

[4] Yu Changjiu, Study on the types of diseases, medical insurance and the selection behavior of medical institutions of farmers, Journal of Agrotechnical Economics, pp.82-92, 2017.

[5] Lu Yijin, Analysis on the influencing factors of elderly patients' choice of medical treatment in China, China Journal of Pharmaceutical Economics, vol.12, pp.18-23+33, 2017.

[6] Zhang Li, Li Wei, Investigation and study on influencing factors of medical treatment behavior of urban and rural residents in Shandong province, Medicine and Philosophy, vol.37, pp.43-46, 2016. 
[7] Cui Huaqian, Fang Guoyu, Yang Yang and Yao Weiguang, Investigation on the knowledge and cognition of community residents in Guangzhou on the hierarchical diagnosis and treatment mode, Chinese General Practice, vol.17, pp.4123-4126, 2014.

[8] Sun Mengjie, Han Huawei, A study on the medical treatment choice of rural residents in China -- an empirical analysis of rural households in Gansu, Henan and Guangdong provinces, Economic Review, pp.40-50+111, 2013.

[9] Xiao Dingding, Zhang Wenfeng, Analysis of key factors of green logistics development based on DEMATEL method, Industrial Engineering, vol.13, pp.52-57, 2010.

[10]Zhang Yan, Tan Ziqiu, Liu Xin and Fan Cuiping, Analysis of influencing factors of patients' choice of hospital, Medicine and Society, vol.23, pp.71-73, 2010.

[11]Qijuan B, Li T, The Study on Medical Care Seeking Behavior of Farmers in Different Income Levels under the New Rural Co-operative Medical System: Based on Data of Farmers in Jiangsu Province, Journal of Yunnan Agricultural University (Social Science), 2015.

[12]Zhang Rongyu, Yin Aitian and An Jian, Research progress on medical behavior and policy influencing factors, China Public Health, vol.28, pp.861-862, 2012.

[13]Pan Zijing, Chai Shenhua, Gao Mengyang and Zhang Xiang, The selection of rural residents' first diagnosis institution and analysis of influencing factors, Medicine and Society,vol.30, pp.8-10+28, 2017.

[14]Bao Ting, Wang Youjuan and Ma Yuanlin, Research progress on residents' medical behavior, Chinese Journal of Social Medicine, vol.36, pp.135-138, 2019.

[15]Liu Ning, Chen Min, Based on the hierarchical diagnosis and treatment perspective of medical behavior analysis and related strategy research, Chinese Hospital Management, vol.36, pp.19-21, 2016. 